\title{
Astroprotein (GFAP) Levels in Cerebrospinal Fluid of Stroke Patients
}

\author{
Toru Hayakawa, Yukitaka Ushio, Yasutaka MaEda, \\ Norio Arita, Toshiki Yoshimine, Mamoru TANEdA*, \\ Tadayoshi IRINo*, Kazuyoshi Morimoto, \\ Takesada Mori** and Heitaro Mogami
}

\begin{abstract}
Departments of Neurosurgery and ${ }^{* *} 2$ nd Surgery, Osaka University Medical School, Osaka; *Division of Cerebrovascular Diseases, Hanwa Memorial Hospital, Osaka
\end{abstract}

\begin{abstract}
Radioimmunoassay was employed to study the astroprotein (Ap) - an astrocyte-specific cerebroprotein which is immunologically identical to glial fibrillary acidic protein (GFAP) - levels in the cerebrospinal fluid (CSF) of stroke patients in the acute or subacute stage. These levels tended to increase in the acute stage, especially in cases with intracerebral hemorrhage. The increases were related to the size of the lesion and the patients' general neurological state. The post-ictal increases were more rapid in cases with intracerebral hemorrhage than with cerebral infarction; both exhibited a correlation between the CSF-Ap levels in the acute stage and the prognosis.
\end{abstract}

Key words: astroprotein, GFAP, cerebroprotein, cerebrospinal fluid, stroke, cerebral hemorrhage

\section{Introduction}

Astroprotein $^{4)}$ (an astrocyte-specific cerebroprotein: Ap) is immunologically identical to glial fibrillary acidic protein (GFAP). " Concentrations of Ap in cerebrospinal fluid (CSF) are frequently known to increase in glioma patients. ${ }^{1,5,6)}$ Our preliminary study $^{2)}$ demonstrated that CSF-Ap increases in stroke patients. Based on these findings, we hypothesized that the appearance and increase of Ap in the CSF may be directly related to degenerative and/or destructive processes in the brain. ${ }^{1,2)}$

In stroke patients, the brain tissue has been acutely destroyed and their prognosis is related to the extent and degree of the lesion. Therefore, to test our hypothesis and to determine the clinical significance of CSF-Ap measurements in stroke patients in the acute stage, we analyzed their CSF-Ap levels by radioimmunoassay.

\section{Materials and Methods}

We obtained $206 \mathrm{CSF}$ samples from 70 stroke patients in the acute or subacute stage. Of these 70 patients, 34 $(49 \%)$ had intracerebral hemorrhage (ICH), $14(20 \%)$ had subarachnoid hemorrhage (SAH), and $22(31 \%)$ had cerebral infarction (INF). Control CSF samples were obtained from 8 patients without clinical or laboratory evidence of intracranial disease. The first CSF samples of 60 stroke patients ( $26 \mathrm{ICH}, 12 \mathrm{SAH}, 22$ INF) were collected during the acute stage, i.e., within 3 days of the ictus, by lumbar puncture. These patients had undergone no neurosurgery prior to the collection of the first CSF samples. In addition, CSF samples were obtained from 37 patients on several occasions during the clinical course. All samples were stored at $-60^{\circ} \mathrm{C}$ until assay.

CSF-Ap was measured by radioimmunoassay as described previously. ${ }^{1,5,6)}$ The present assay method can detect Ap concentrations ranging from 5 to

Received February 24, 1983; Accepted June 8, 1983

Author's present address: T. Irino, M. D., Department of Traumatology, Osaka University Medical School, Osaka, Japan. 
$1,000 \mathrm{ng} / \mathrm{ml}$. The Ap concentration of all CSF samples was determined; the total protein level was measured in 115 of the 206 CSF samples.

\section{Results}

Figure 1 shows the CSF-Ap concentration in 60 stroke patients in the acute stage. The mean CSF-Ap value in the control group was $10.0 \pm 1.07 \mathrm{ng} / \mathrm{ml}$; values above $25 \mathrm{ng} / \mathrm{ml}$ were considered abnormal. According to this criteria, 24 of $26(92.3 \%)$ patients with $\mathrm{ICH}, 7$ of 12 $(58.3 \%)$ patients with $\mathrm{SAH}$, and 15 of $22(68.2 \%)$ patients with INF manifested abnormally high CSFAp levels. CSF-Ap was above $1,000 \mathrm{ng} / \mathrm{ml}$ in 14 of 26 $(53.8 \%) \mathrm{ICH}, 2$ of $12(16.7 \%) \mathrm{SAH}$, and 6 of $22(27.3 \%)$ INF cases. Differences between the CSF-Ap levels of the control and each stroke group were statistically significant (each $\mathrm{P}<0.05$ ).

Figure 2 shows the relationship between CSF-Ap levels and the size and location of the hematomas with or without ventricular rupture in the 26 patients with ICH. Although there was no definite relationship between the level of CSF-Ap and the location of the hematoma, patients with large hematomas or ventricular rupture tended to manifest higher CSF-Ap levels.

In the 12 stroke patients with $\mathrm{SAH}$, we noted a relationship between the CSF-Ap level and the clinical grade of SAH classified according to Hunt and Kosnik $(1974)^{3 \prime}$ (Fig. 3). Patients with higher grades of SAH

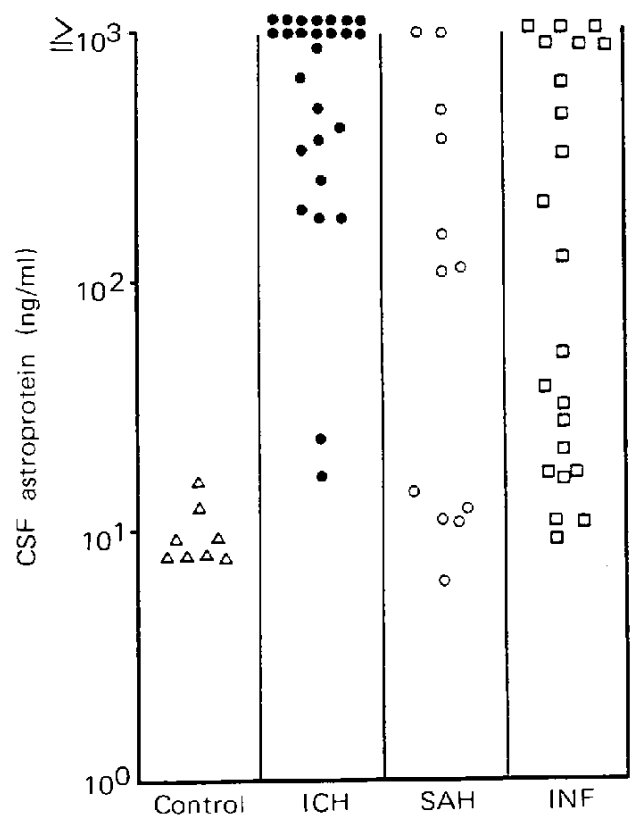

Fig. 1 CSF astroprotein levels in 60 stroke patients in the acute stage. ICH: intracerebral hemorrhage $(\mathbf{n}=26)$, SAH : subarachnoid hemorrhage $(n=12)$, INF : cerebral infarction $(n=22)$.

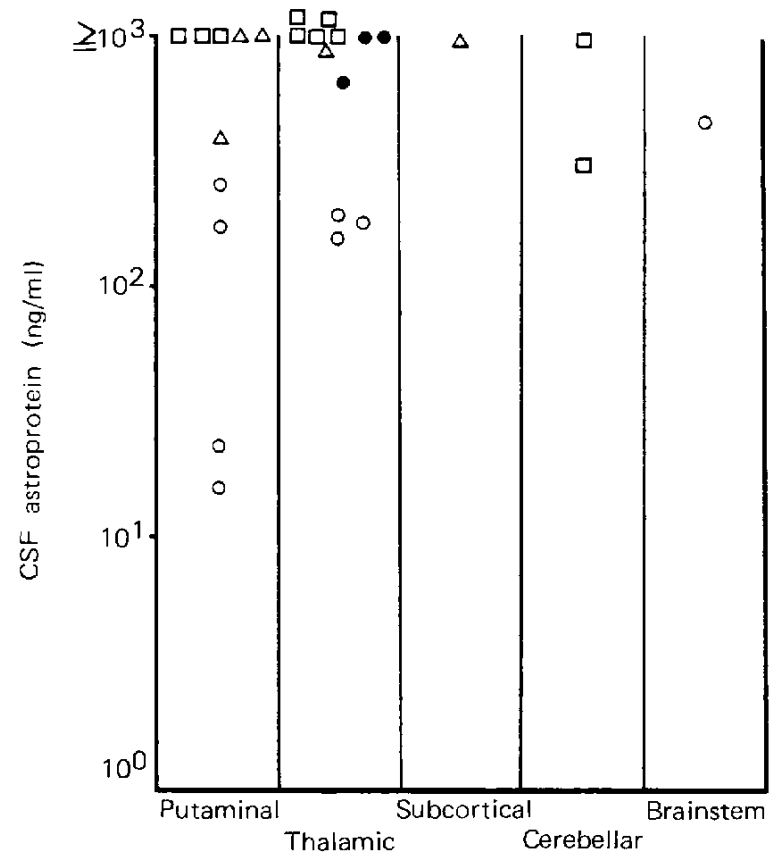

Fig. 2 Relationship between CSF astroprotein levels and the size and location of the hematoma. Assessment was of 26 stroke patients with intracerebral hemorrhage during the acute stage. Small hematoma with $(O)(n=3)$ and without $(O)(n=8)$ ventricular rupture; large hematoma with $(\square)$ (n $=10)$ and without $(\Delta)(n=5)$ ventricular rupture.

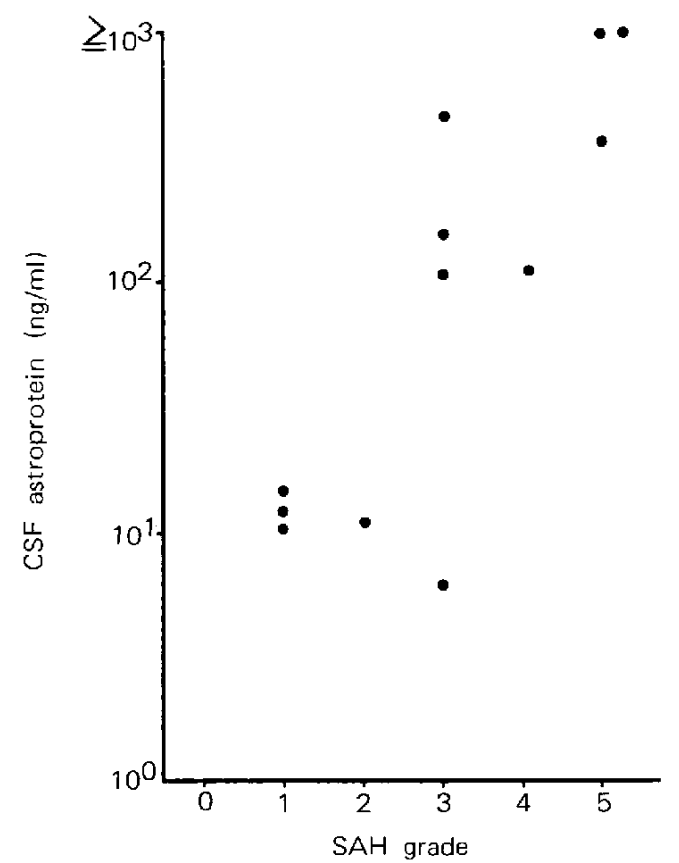

Fig. 3 Relationship between CSF astroptotein levels and the clinical grade of subarachnoid hemorrhage, classified according to Hunt and Kosnik ${ }^{3)}$ $(\mathrm{n}=12)$.

Neurol Med Chir (Tokyo) 24, January, 1984 
manifested higher CSF-Ap levels. There was also a relationship between the CSF-Ap level and the level of consciousness in the 22 stroke patients with INF (Fig. 4). Patients with slighter disturbances of consciousness tended to show lower CSF-Ap levels.

Figure 5 shows the post-ictal changes in the CSF-Ap levels of patients with ICH. In most cases the level of CSF-Ap was high on the day of the ictus, remained elevated for several days, and then decreased gradually.

We divided the post-ictal changes in the CSF-Ap level of patients with INF into 2 categories. In category 1, CSF-Ap increased sharply soon after the occurrence of the ictus, although in comparison to stroke patients with $\mathrm{ICH}$, the increase was somewhat delayed. In category 2 , the increase in CSF-Ap began later than in category 1 , was classified as slight or moderate, and peaked at around the 5th post-ictal day. Furthermore, patients who manifested category 1 changes could be separated into 2 groups; those with large infarctions who died within a few days of the ictus and those who were confirmed by CT scan or cerebral angiography to have hemorrhagic infarction or recanalization of the obstructed arteries. In the latter group, the CSF-Ap level decreased approximately one week after the ictus (Fig. 6).

Our data on the CSF-Ap changes in stroke patients with SAH were insufficient to draw any conclusion.

Figure 7 shows the relationship between the CSF-Ap levels during the acute stage of stroke and the clinical

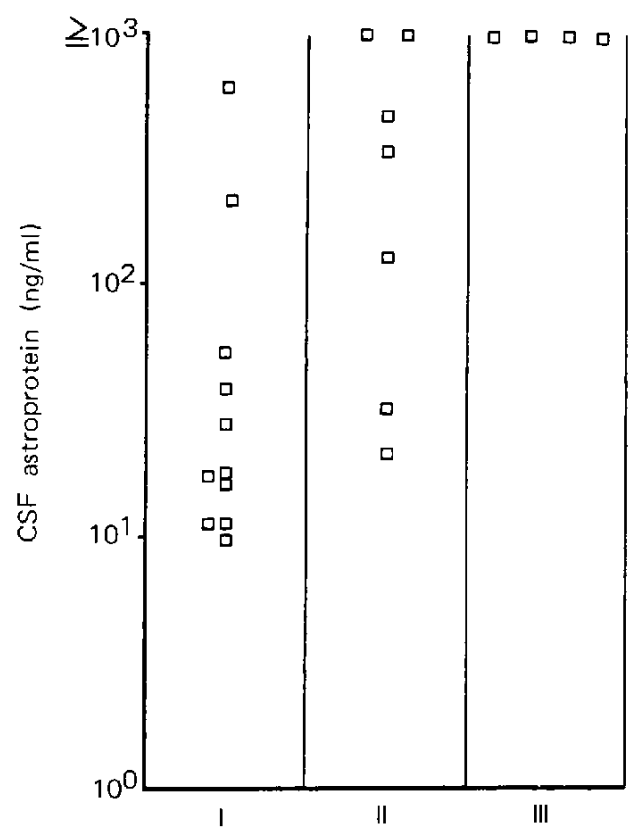

Fig. 4 Relationship between CSF astroprotein levels and the level of consciousness in 22 stroke patients with cerebral infarction. I: confusion $(\mathbf{n}=11)$, II : stupor $(\mathrm{n}=7)$, III : coma $(n=4)$.

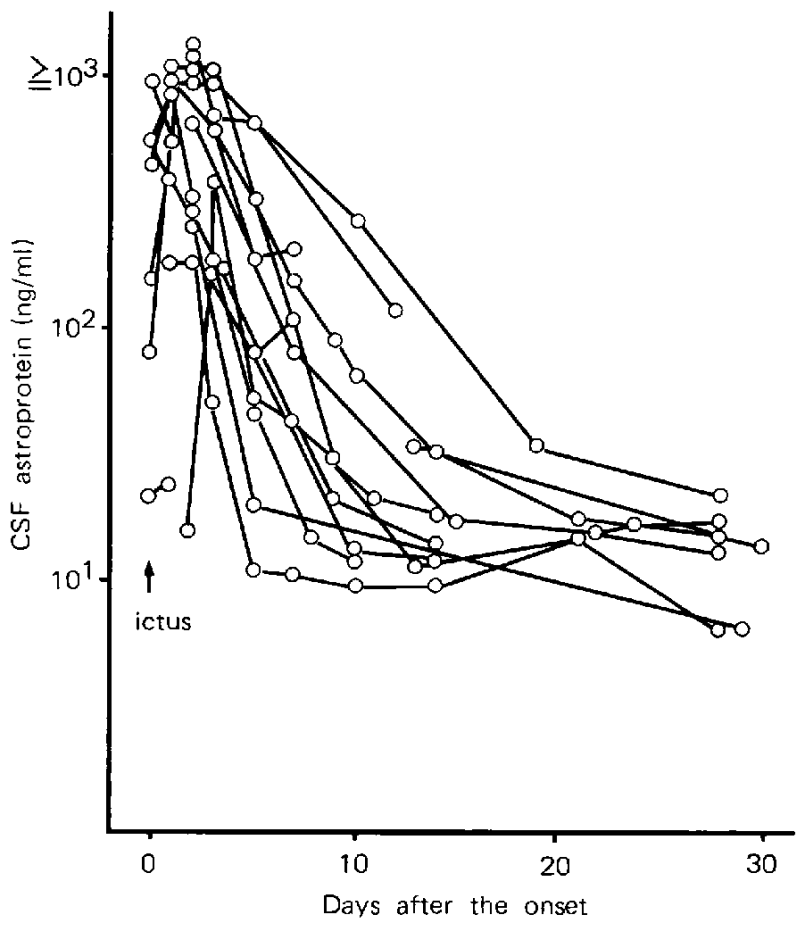

Fig. 5 Changes in the CSF astroprotein in stroke patients with intracerebral hemorrhage $(n=13)$.

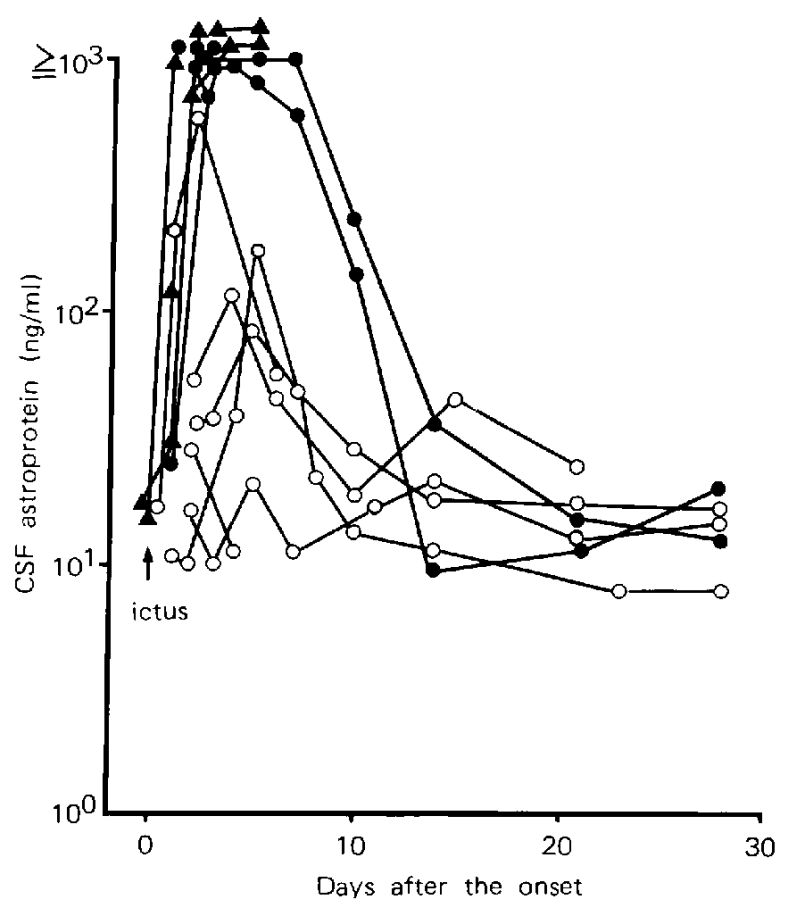

Fig. 6 Changes in the CSF astroprotein levels in stroke patients with cerebral infarction $(n=12) . \quad \mathbf{A}$ : large infarction, resulting in death $(n=3)$, hemorrhagic infarction or recanalization of the obstructed arteries $(n=3)$, 0 : infarction of moderate size $(n=6)$. 


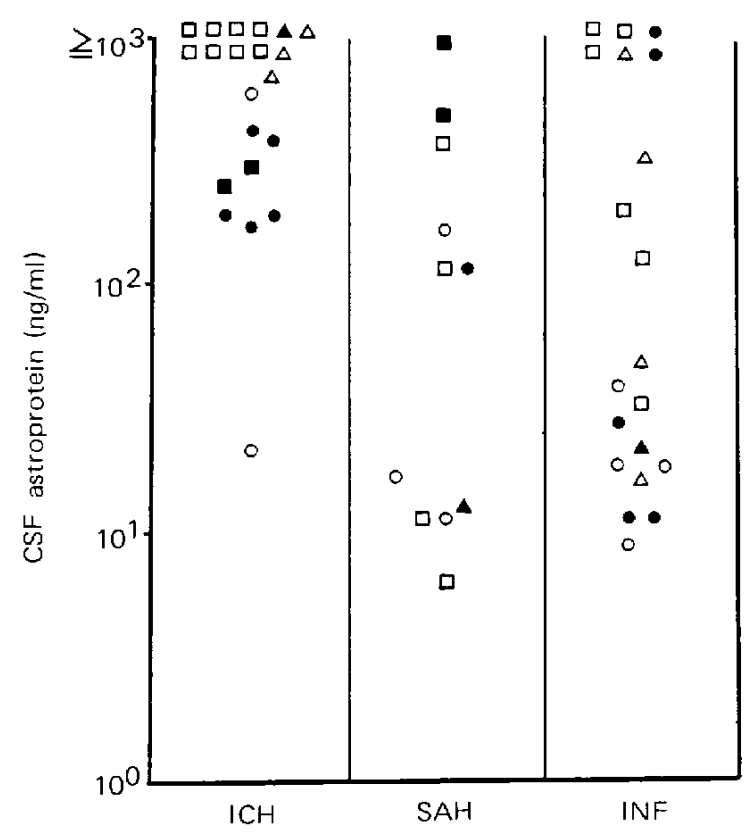

Fig. 7 Relationship between the CSF astroprotein level in 52 stroke patients in the acute stage and clinical outcome. $\bigcirc$ :able to do active work $(\mathbf{n}=9)$, able to care for self $(n=11), \triangle$ : able to live at home with assistance $(\mathbf{n}=7), \mathbf{\Delta}$ : unable to care for self $(\mathbf{n}=3), \square:$ died $(\mathrm{n}=18), \mathbf{\square}$ : died due to complications $(n=4)$.

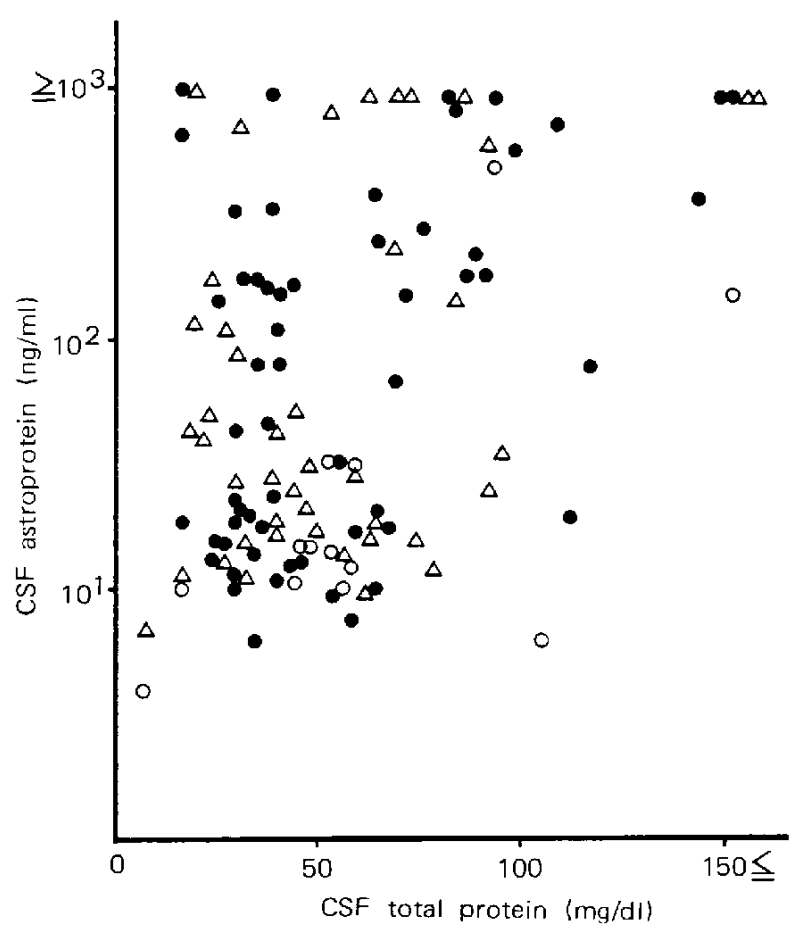

Fig. 8 Relationship between CSF astroprotein and total protein levels in stroke patients. intracerebral hemorrhage $(\mathrm{n}=58), \quad \bigcirc$ : subarachnoid hemorrhage $(\mathbf{n}=13), \triangle$ : cerebral infarction $(n=40)$. outcome for 52 patients. Among patients with $\mathrm{ICH}$, those who manifested lower CSF-Ap levels during the acute stage showed a better prognosis. Of 20 patients with INF, $6(30 \%)$ died, 3 of whom had shown a marked increase in CSF-Ap (above $1,000 \mathrm{ng} / \mathrm{ml}$ ) during the acute stage. The CSF samples of the other 3 patients which showed a slight to moderate increase, were obtained on, or one day after, the day of the ictus. In stroke patients with $\mathrm{SAH}$, there was no definite relationship between the CSF-Ap level during the acute stage and the prognosis.

Figure 8 shows that there is no statistically significant correlation between the CSF-Ap and CSF total protein levels in stroke patients with $\mathrm{ICH}, \mathrm{SAH}$, or INF.

\section{Discussion}

Our study confirmed that the CSF-Ap level increased in stroke patients, particularly in those with ICH, during the acute stage. Though there was no apparent relationship between the location of hematoma and the CSF-Ap level, our findings demonstrated that patients with large hematomas and those with ventricular rupture manifested more marked increases in CSF-Ap (Fig. 2). A relationship was observed between the CSFAp level and the clinical grade of SAH in patients with SAH (Fig. 3) and the level of consciousness in patients with INF (Fig. 4). Our findings support the hypothesis that the increase in CSF-Ap is directly related to the extent and degree of the destructive and/or degenerative processes in the brains of stroke patients.

As shown in Fig. 2, if there was ventricular rupture in patients with ICH, the CSF-Ap levels tended to be higher, irrespective of the size of hematoma. The direct communication between the lesion and the CSF pathway and/or the rich distribution of fibrillary astrocytes in the subependimal region may be responsible for this result.

In patients with $\mathrm{ICH}$, the CSF-Ap level increased remarkably immediately upon the occurrence of the ictus (Fig. 5). On the other hand, this increase was somewhat delayed in patients with INF, even in the presence of large infarctions (Fig. 6). Our unpublished data suggest that the turn-over rate of Ap in CSF may be very high. It is possible that the CSFAp level may be indicative of the extent and degree of the destructive and/or degenerative processes in progress at the time of CSF sampling. Our present findings on the post-ictal changes in CSF-Ap suggest that fundamentally different destructive and/or degenerative processes may take place in patients with ICH, SAH, and INF.

Interestingly there appeared to be three types of 
changes in the CSF-Ap level of patients with INF. In cases with large infarctions which led to death, the CSF-Ap levels increased markedly upon the occurrence of the ictus. In cases with confirmed hemorrhagic infarction or recanalization of the obstructed arteries, the increase was also rapid and pronounced, and followed by a gradual decrease which started about one week after the ictus. In cases with moderate infarctions, the increase in CSF-Ap was delayed and slight to moderate in degree. In patients with large infarctions, brain destruction may be rapid and fatal although its inception may be somewhat later than in patients with ICH. On the other hand, in cases with moderate infarctions, the destructive and/or degenerative processes may peak somewhat later than in patients with large infarctions and in the early stage of these processes, the majority of the lesions may be reversible. The destruction may be rapid in patients with hemorrhagic infarctions, as in patients with $\mathrm{ICH}$, though the onset may be delayed for a few days after occurrence of the infarction.

Good correlation was observed between CSF-Ap levels during the acute stage in patients with $\mathrm{ICH}$ and their prognosis (Fig. 7). Of the patients with INF, 6 died. Three manifested a marked increase in CSF-Ap while the other 3 showed only a slight to moderate increase. CSF samples were collected on the day of, or the day following, the ictus in the latter patients. Considering the changes in CSF-Ap in patients with INF, a better correlation between the CSF-Ap level and the prognosis may be obtained if the CSF samples were collected later, e.g. 3-5 days after the ictus. Those patients with INF who were confirmed to have hemorrhagic infarctions or recanalization of the obstructed arteries manifested a relatively good clinical outcome, although their CSF-Ap levels in the acute stage had been markedly high. This result is of interest from the viewpoint of the pathogenesis of hemorrhagic infarction.

The correlation between the CSF-Ap levels and the prognosis was poor in patients with SAH. The intracranial aneurysms in most of these patients were treated neurosurgically. It is possible that aneurysmal re-rupture or the occurrence of post-sampling vasospasms may have affected the clinical outcome. In addition, the prognosis may have been affected by the surgical procedures.

There was no statistical correlation between the CSF-Ap and CSF total protein levels (Fig. 8), suggesting that the determination of CSF-Ap may have a significance which is different from that of CSF total protein. The result may also indicate that CSF-Ap levels do not have direct correlation with the volume of blood spilled in CSF at time of ictus.
Our findings suggest that the CSF-Ap levels in stroke patients may be indicative of the extent and degree of degenerative and/or destructive processes and that determination of CSF-Ap in the acute stage may aid in drawing a prognosis, especially in patients with $\mathrm{ICH}$ and INF. The usefulness of CT scanning in the diagnosis of stroke patients has already been established. To evaluate the clinical value of CSF-Ap measurement, further studies which determine the advantage or superiority of this method over CT scanning are needed. Potentially, CSF-Ap determination may possibly facilitate the differential diagnosis between reversible edematous and irreversible degenerative and/or destructive lesions, both of which appear as similar low density areas on CT scans.

\section{Conclusions}

1. Concentrations of astroprotein (GFAP) in cerebrospinal fluid of stroke patients frequently increased during the acute or subacute stage.

2. To measure of cerebrospinal fluid levels of astroprotein (GFAP) may be useful in determining the extent and degree of destructive and/or degenerative processes in the brain.

\section{Acknowledgments}

The authors wish to thank the Department of Neurosurgery, Osaka University Medical School, the Department of Neurosurgery, the Center for Adult Diseases, and the Division of Cerebrovascular Diseases, Hanwa Memorial Hospital, for providing CSF samples. We are also grateful to Dainabot Radioisotope Laboratories for the astroprotein radioimmunoassay.

\section{References}

1) Hayakawa $T$, Morimoto $\mathrm{K}$, Ushio $\mathrm{Y}$, Mori $\mathrm{T}$, Yoshimine T, Myoga A, Mogami $\mathbf{H}$ : Levels of astroprotein (an astrocyte-specific cerebroprotein) in cerebrospinal fluid of patients with brain tumors. An attempt at immunochemical diagnosis of gliomas. $J$ Neurosurg 52: $229-233,1980$

2) Hayakawa $T$, Ushio $Y$, Mori $T$, Arita N, Yoshimine $T$, Maeda Y, Shimizu K, Myoga A: Levels in stroke patients of CSF astroprotein. Stroke 10: 685-689, 1979

3) Hunt WE, Kosnik EJ: Timing and preoperative care in intracranial aneurysm surgery. Clin Neurosurg 21: $78-79,1974$

4) Mori T: Studies on astrocyte-specific antigen (astroprotein). Neurochemistry (Tokyo) 9: 75-78, 1970 (in Japanese)

5) Mori T, Morimoto K, Hayakawa T, Ushio Y, Mogami $\mathrm{H}$, Sekiguchi $\mathrm{K}$ : Radioimmunoassay of astroprotein 
(an astrocyte-specific cerebroprotein) in cerebrospinal fluid and its clinical significance. Neurol Med Chir (Tokyo) 18 [Part I]: 25-31, 1978

6) Mori T, Morimoto K, Ushio Y, Hayakawa T, Mogami $\mathrm{H}$ : Radioimmunoassay of astroprotein (an astrocytespecific cerebroprotein) in cerebrospinal fluid from patients with glioma. A preliminary study. Neurol Med Chir (Tokyo) 15 [Part I]: 23-25, 1975

7) Mori T, Morimoto K, Ushio Y, Mogami H: Studies on the identity of astroprotein (Mori) and glial fibrillary acidic protein (Eng). Igaku-no-ayumi 92: 16-17, 1975 (in Japanese)

Address reprint requests to $:$ T. Hayakawa, M. D., Department of Neurosurgery, Osaka University Medical School, 1-1-50 Fukushima, Fukushima-ku, Osaka 553, Japan. 